\title{
Evaluation of dry eye disease in newly diagnosed anxiety and depression patients using anterior segment optical coherence tomography
}

Mahmut Oğuz Ulusoy ${ }^{1 *}$ (D), Selen Işık-Ulusoy ${ }^{2}$ and Sertaç Argun Kıvanç3

\begin{abstract}
Background: We aimed to evaluate dry eye diseases (DED) in patients with newly diagnosed depression and anxiety patients.

Methods: Forty newly diagnosed depression, 35 anxiety patients, and 37 controls without any history of taking psychiatric drugs (or before the beginning of psychiatric medication) and topical ophthalmic drop use, were included in the study. All depression and anxiety diagnoses were performed by an experienced psychiatrist. Beck depression and anxiety tests were used to measure disease severity. Tear film break up time (TBUT), Schirmer's test, Oxford scores and ocular surface disease index (OSDI) were admiinistered to participants. Anterior segment optical coherence tomography was used to measure tear meniscus heights (TMH), tear meniscus depths (TMD) and tear meniscus areas (TMA).
\end{abstract}

Results: In anxiety and depression groups, Schirmer's test $(\mathrm{mm})(7.24 \pm 6.02,6.58 \pm 4.9$ and $18.79 \pm 4.9$ respectively, $p<0.05)$ and TBUT (s) $(5.62 \pm 3.1,5.6 \pm 3.5$ and $13.37 \pm 1.7$ respectively, $p<0.05)$ were significantly lower than control group. In addition, OSDI and Oxford scores were significantly higher than controls. OSDI scores were $28.01 \pm 19,30.43 \pm$ $18.49,14.38 \pm 8.14$ respectively $(p=0.002)$ and Oxford scores were $1.9 \pm 0.7,2.1 \pm 0.6$ and $0.7 \pm 0.4$ respectively $(p=0.001)$. TMD, TMH and TMA values were significantly lower in anxiety and depression groups compared with control groups. Correlations between disease inventory scores and dry eye tests were detected.

Conclusions: This study showed a relation between DED and newly diagnosed anxiety and depression patients with no history of psychiatric drug use. The presence of correlation between dry eye tests and disease inventory scores strengthens this association. This is an important knowledge that need to be evaluated in these patients before starting psychiatric medication.

Keywords: Depression, Anxiety, Dry eye, Tear meniscus, Anterior segment optical coherence tomography

\section{Background}

Dry eye disease (DED) is a common disease that affects the ocular surface with reported prevalence rates changes from 11 to $54 \%$. [1] It is a multifactorial disease of the tear film and ocular surface that results in symptoms of discomfort such as pain, heaviness, grittiness, burning, dryness, itchiness, foreign body sensation, visual disturbances, and tear

\footnotetext{
* Correspondence: drmoguz@gmail.com

This manuscript was presented in ESCRS 2017, Lisbon as a free paper. ${ }^{1}$ Department of Ophthalmology, Başkent University School of Medicine Konya Research Hospital, 42000 Konya, Turkey

Full list of author information is available at the end of the article
}

film instability according to the International Dry Eye Workshop. [2] Depression is associated with comorbidities such as hypothyroidism, systemic lupus erythematosus, myasthenia gravis, liver diseases, hypertension, diabetes mellitus, cardiac disease, gastrointestinal disorders, rheumatic diseases, and systemic medications. [1, 3, 4] In addition, an association between DED and depression or anxiety treatment was reported in several studies. [5-7] The prevalence of DED symptoms in depressive and anxiety patients ranged from 21 to $52 \%$ in previous studies. [8-10] However, the main cause of DED in those patients is uncertain, whether due to illness, medication or both. 
The aim of this study was to evaluate the ocular surface and anterior segment optical coherence tomography (AS-OCT) findings in patients who had depression and anxiety disorders, but without any history of psychiatric drug use.

\section{Methods}

\section{Design of Study Groups}

This study was conducted at Başkent University School of Medicine, Konya Research Hospital, Department of Ophthalmology, and approved by the Institutional Review Board and Ethics Committee of the same University (Project No: KA17/106). This study adhered to the tenets of the Declaration of Helsinki and informed consent was obtained from all patients. Forty newly diagnosed depressive disorder patients, 35 newly diagnosed anxiety disorder patient and 37 healthy control patients were recruited in this study. Newly diagnosed anxiety and depression patients were referred to the ophthalmology department immediately after diagnois before initiation of psychiatric medication. Patients without any history of dry eye, and systemic and topical drug use, were selected for the study.

\section{Psychiatric diagnoses}

The Structured Clinical Interview for Diagnostic and Statistical Manual of Mental Disorders-IV (DSM-IV) Axis I disorders (SCID-I) was used for psychiatric disorders. [11] The interview was done by an experienced psychiatrist that was unaware of ocular findings and scores on all self-reported questionnaires (Beck Depression Index (BDI) and Beck Anxiety Inventory (BAI)).

BDI and BAI were used for evaluating patient mental health. The questionnaire consists of 21 items that scale, from 0 (neutral) to 3 (maximum intensity), and the final score range was from 0 to 63 points.

For BAI scores were divided into four degrees due to disease severity. (0 to 7 indicates normal range, 8-15 mild anxiety, 16-25 moderate anxiety and 26-63 severe anxiety). [12] Overall reliability of the scales which was adapted to Turkish version computed by Cronbach's alfa was 0.90 .

For BDI scores of 0-9 are normal, scores of 10-16 indicate mild depression, scores of 17-29 indicate moderate depression and scores of 26-63 indicate severe symptoms of depression. [13]

Psychiatric exclusion criteria from the study were previous medication for anxiety or depressive disorder, a history of seizure disorder, serious or unstable medical illness, substance abuse disorder (active within the past year), serious suicidal risk, schizophrenia, or major antisocial personality disorder.

After psychiatric diseases were diagnosed, patients were referred to the Ophthalmology Department.

\section{Ophthalmic examinations}

Detailed full ophthalmic examination was done for all patients such as visual acuity (by Snellen chart), refraction assessment, biomicroscopic examination, intraocular pressure measurement and fundus examination.

Patients completed the ocular surface disease index (OSDI) at initial visit. [8] AS-OCT tear meniscus parameters, Schirmer, tear break-up time (TBUT) and corneal staining scores (Oxford Scheme) were measured for all patients. [14]

A spectral domain optical coherence system (RTVue100; Optovue, Fremont, CA) with a corneal adaptor module was used. This system has a $6-\mathrm{mm}$ vertical beam that takes 26,000 axial scans per second and has a 5-mm axial resolution to a depth of $2.8 \mathrm{~mm}$. Vertical images were recorded at the 6-o'clock position of the cornea $3 \mathrm{~s}$ after each blink, which was repeated 3 times, and a built-in calliper was used to measure tear meniscus heights (TMH), tear meniscus depths (TMD) and tear meniscus areas (TMA). The mean of the 3 measurements was used for analysis. TMH was determined as the length from the point where the meniscus intersected with the cornea superiorly to the eyelid inferiorly. TMD was determined as the length from the apex of the fornix to the surface of the tear meniscus, perpendicular to $\mathrm{TMH}$. The borders of the tear meniscus were marked with a calliper, and integrated analysis software calculated the area in $\mathrm{mm}^{2}$ to measure TMA. Only measurements of the right eye were used for statistical analysis. (Fig. 1).

Corneal staining scores were classified as 0 to 5 based on the Oxford Scheme. [14] Finally, Schirmer test was performed for a duration of 5 min without topical anaesthetic drops. The filter paper strip was placed in the middle and lateral thirds of the lower eyelid.

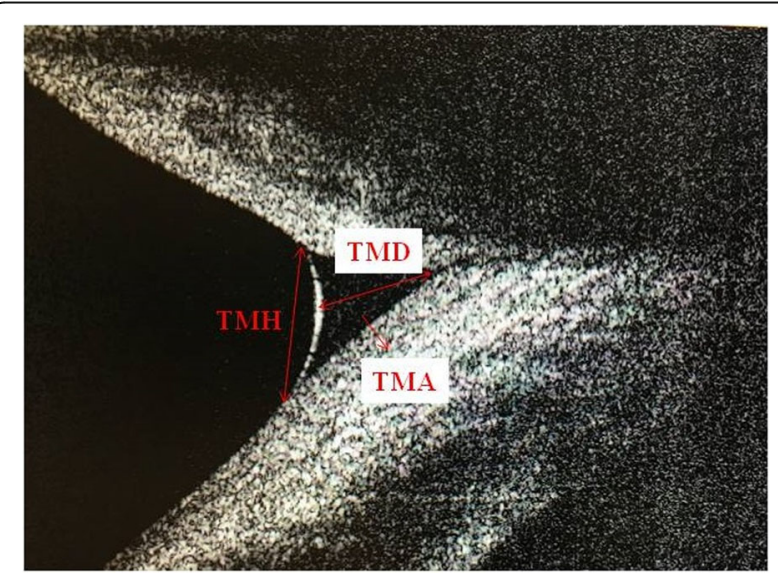

Fig. 1 Image of measurement of tear meniscus height, tear meniscus depth and tear meniscus area using anterior segmentoptical coherence tomography 
Table 1 Demographic factors, disease severity index and conventional dry eye test results for all groups

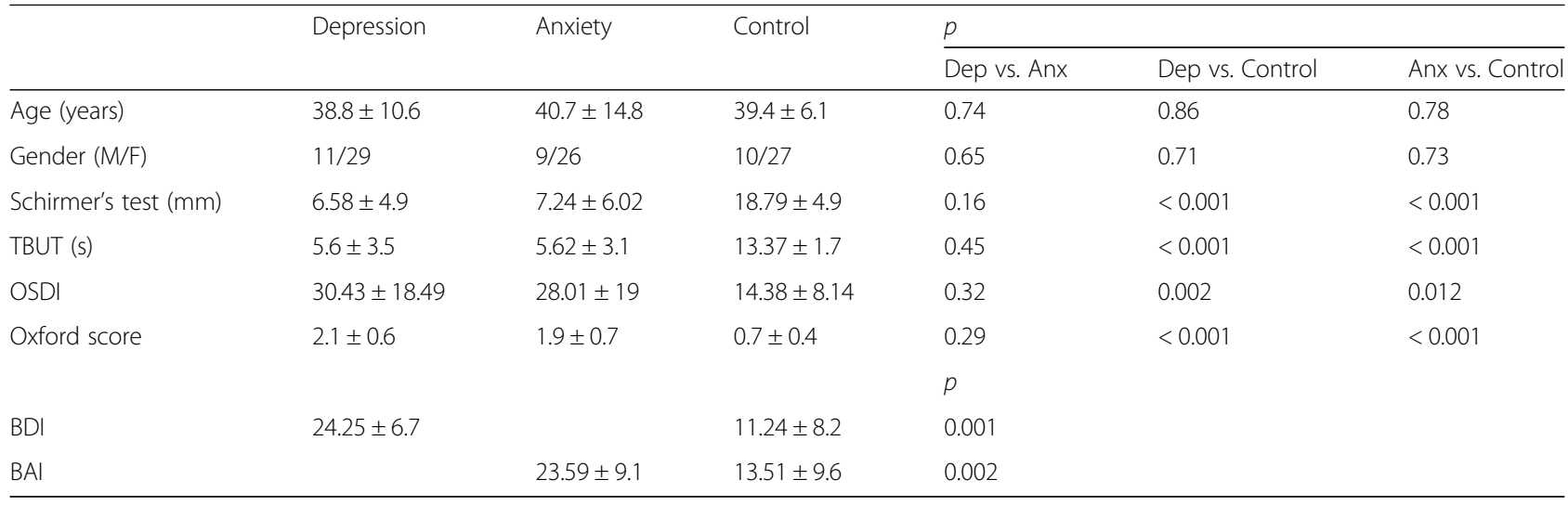

$M=$ male, $F=$ female, $B D I=$ Beck depression inventory, $B A I=$ Beck anxiety inventory, $T B U T=$ tear break up time, $O S D I=$ ocular surface disease index

\section{Dry eye diagnosis}

Schirmer's test was used to quantify the tear secretion within 5 min by wetting of the filter paper placed on the ocular surface. Schirmer $<10 \mathrm{~mm}$ in the unanaesthetized eye is considered to be consistent with a disturbance in the tear film. TBUT was measured after impregnated of a moistened fluorescein 1-mg strip in the lateral onethird of the patient's lower eyelid. The interval between the last complete blink and the appearance of the first corneal black spot in the stained tear film was measured. TBUT is accepted as evidence for dry eye, if it is shorter than $10 \mathrm{~s}$. The Oxford scoring system is as follows with $1=$ mild, 2 = moderate, and scores of $>3$ being consist ent with severe DED.

Patients with a history of ocular surface diseases and inflammation, contact lens wearing, any ocular surgery, and any systemic disease that could affect measurements were excluded. Also use of any eye medications or artificial tears during the previous month were excluded.

\section{Statistical analysis}

SPSS version 21.0 (SPSS, Chicago, IL, USA) was used for statistical analysis. The normality of the values was analysed using the Kolmogorov-Smirnov test. One-way ANOVA test followed by Tukey's post hoc was used to analyse the difference between the three groups. Correlations between the variables were investigated using the Pearson or Spearman correlation coefficient. $p<0.05$ was considered as significant.

\section{Results}

The average age in years did not differ significantly between the three groups $(38.8 \pm 10.6$ in the depression group, $40.7 \pm 14.8$ in the anxiety group and $39.4 \pm 6.1$ in the control group, $p=0.765$ ). Male-to-female ratio is $11 /$ 29 in the depression group, $9 / 26$ in the anxiety group and 10/27 in control group $(p=0.658)$. Mean BDI of depression and control groups were $24.25 \pm 6.7$ and $11.24 \pm 8.2$, respectively, $(p=0.001)$. Mean BAI of anxiety and control groups were $23.59 \pm 9.1$ and $13.51 \pm 9.6$, respectively, $(p=0.002)$.

Conventional dry eye tests were significantly different in depression and anxiety groups compared with the control group. As expected Schirmer test and TBUT were lower and OSDI and corneal staining scores were higher in the patient groups than in controls. $(p<0.05$ for Schirmer test, TBUT, OSDI and corneal staining scores). Tear meniscus parameters for the depression and anxiety groups were significantly lower compared with the control group ( $p<0.05$ for TMH, TMD, and TMA). Demographic characteristics, Schirmer, TBUT, OSDI, staining scores, tear meniscus parameters and psychiatric test scores are listed in Tables 1 and 2.

In the depression group, Schirmer's test was significantly correlated with all tear meniscus parameters; TBUT was correlated with TMH and TMA, OSDI and corneal staining scores were correlated with only TMA (Table 3). However, in the anxiety group, while Schirmer test was significantly correlated with all tear meniscus

Table 2 Comparison of AS-OCT parameters among depression, anxiety and control groups

\begin{tabular}{lllllll}
\hline & Depression & Anxiety & Control & $p$ & & \\
\cline { 5 - 7 } & & & & Dep vs. Anx & Dep vs. Control & Anx vs. Control \\
\hline TMH $(\mu \mathrm{m})$ & $215.28 \pm 55.7$ & $198.82 \pm 48.09$ & $465.29 \pm 183.25$ & 0.81 & $<0.001$ & $<0.001$ \\
TMD $(\mu \mathrm{m})$ & $149.1 \pm 35.41$ & $125.68 \pm 29.72$ & $260.97 \pm 91.06$ & 0.21 & $<0.001$ & $<0.001$ \\
TMA $\left(\mathrm{mm}^{2}\right)$ & $0.01 \pm 0.005$ & $0.01 \pm 0.004$ & $0.11 \pm 0.02$ & 0.97 & $<0.001$ & $<0.001$ \\
\hline
\end{tabular}

$A S-O C T=$ anterior segment optical coherence tomography, $T M H=$ tear meniscus height, $T M D=$ tear meniscus depth, $T M A=$ tear meniscus area 
Table 3 Correlation between AS-OCT parameters and conventional dry eye tests in patients with depression

\begin{tabular}{|c|c|c|c|c|c|c|}
\hline \multirow{3}{*}{ Conventional Tests } & \multicolumn{6}{|c|}{ AS-OCT PARAMETERS } \\
\hline & \multicolumn{2}{|l|}{$\mathrm{TMH}$} & \multicolumn{2}{|l|}{ TMD } & \multicolumn{2}{|l|}{ TMA } \\
\hline & $r$ & $p$ & $r$ & $\mathrm{p}$ & $r$ & $P$ \\
\hline Schirmer's test (mm) & 0.479 & 0.01 & 0.405 & 0.002 & 0.548 & $<0.001$ \\
\hline TBUT (s) & 0.405 & 0.012 & 0.287 & 0.81 & 0.392 & 0.015 \\
\hline Oxford Score & -0.189 & 0.35 & -0.361 & 0.26 & -0.425 & 0.032 \\
\hline OSDI & -0.256 & 0.11 & -0.254 & 0.11 & -0.318 & 0.045 \\
\hline
\end{tabular}

$A S-O C T=$ anterior segment optical coherence tomography, $T B U T=$ tear break up time, $O S D I=$ ocular surface disease index, $T M H=$ tear meniscus height, $T M D=$ tear meniscus depth, $T M A=$ tear meniscus area

parameters, TBUT was correlated with TMD and TMA, OSDI was correlated with TMH and TMA parameters (Table 4).

BAI score was correlated with TBUT $(r=-0.423$, $p=0.013)$ in patients with anxiety, and BDI was correlated with TBUT $(\mathrm{r}=-0.343, p=0.035)$, TMD $(\mathrm{r}=$ $-0.45, p=0.004)$, TMH $(\mathrm{r}=-0.448, p=0.004)$ and TMA $(\mathrm{r}=-0.454, \quad p=0.003)$ in patients with depression.

\section{Discussion}

Depression and anxiety are associated with DED, however, there is not enough evidence that have evaluated the effects of anxiety and depression to DED. [5] Tiskaoğlu et al. reported that newly diagnosed depressive disorder patients were associated with DED. [15] They suggested that disturbances of the serotonin receptors, which are located around the conjunctival epithelium, can influence meibomian glands, and this can lead to tear film deficiency in depression patients. [16] Consistent with this hypothesis, a recent study reported that patients with DED have higher levels of serotonin in their tears compared with individuals without DED. [17] In the same study, patients with DED symptoms with normal tear production had similar serotonin levels with patients without DED symptoms. [17] These patients correspond to patients with chronic ocular pain TBUT without any defect on tear film parameters. [17] The authors have explained this situation

Table 4 Correlation between AS-OCT parameters and conventional dry eye tests in patients with anxiety

\begin{tabular}{|c|c|c|c|c|c|c|}
\hline \multirow[t]{3}{*}{ Conventional Tests } & \multicolumn{6}{|c|}{ AS-OCT PARAMETERS } \\
\hline & \multicolumn{2}{|l|}{$\overline{\mathrm{TMH}}$} & \multicolumn{2}{|l|}{ TMD } & \multicolumn{2}{|l|}{ TMA } \\
\hline & $r$ & $p$ & $r$ & $p$ & $r$ & $P$ \\
\hline Schirmer's test $(\mathrm{mm})$ & 0.622 & 0.02 & 0.538 & 0.03 & 0.778 & 0.01 \\
\hline TBUT (s) & 0.182 & 0.3 & 0.674 & 0.01 & 0.682 & 0.03 \\
\hline Oxford Score & -0.219 & 0.27 & -0.251 & 0.45 & -0.487 & 0.32 \\
\hline OSDI & -0.646 & $<0.05$ & -0.184 & 0.64 & -0.533 & 0.018 \\
\hline
\end{tabular}

$A S-O C T=$ anterior segment optical coherence tomography, $T B U T=$ tear break up time, $O S D I=$ ocular surface disease index, $T M H=$ tear meniscus height, $T M D=$ tear meniscus depth, $T M A=$ tear meniscus area as these patients may have developed central sensitization with DED-associated neuropathic pain e.g. chronic pain symptoms. [17] Others suggested that chronic pain is a potent stress factor that affects mood and have association with mood disorders like depression and anxiety. [18] Chronic pain due to DED, may cause or aggravate the symptoms of these mood disorders. In addition, depression and pain use same biological pathways and neurotransmitters such as adrenaline and serotonin. [19, 20] According to these information, a study became noteworthy which showed the decreased platelet serotonin levels in patients with primary Sjögren's syndrome akin to depression patients. [21] Any disturbance in meibomian glands may lead to irregularity in secretion of the lipid layer and thus may explain the correlation of short TBUT with depression and anxiety scores. Therefore, these results suggest that, there can be a pathophysiologic intersection between DED and mood disorders.

Our study showed an association between DED and newly diagnosed depression and anxiety. Schirmer's test, TBUT, OSDI and corneal staining scores were significantly different in the depression and anxiety groups compared with control group. In a similar report, Tiskaoğlu et al. showed an association between DED and newly diagnosed depressive disorder. [15] However, in this study, our patients' Schirmer and TBUT results were lower while, OSDI scores and corneal staining scores were similar.

One strongest part of this study was, our study was designed with newly diagnosed patients without any systemic psychiatric drug (before beginning of psychiatric medication) and/or ocular drop usage. Previous studies, which reported association between DED and depression and anxiety, evaluated patients that already use antidepressant and antianxiety treatment. [1, 22-24] Therefore, it became very different to discriminate whether DED was caused from medical treatment or illness itself. Results from a previous study, showed a correlation between DED and duration of psychiatric disease, selective serotonin reuptake inhibitors (SSRIs) use and duration of SSRIs use, which supports that these reports did not find out the own effect of diseases. [1] Furthermore, we evaluated newly diagnosed anxiety patients in our study. Similarly, anxiety patients' dry eye parameters were significantly different from control group. Another novel part of this study was usage of anterior segment OCT in depression and anxiety patients.

We also evaluated using AS-OCT tear meniscus parameters of newly diagnosed depressive and anxiety disorder patients. The AS-OCT is a non-invasive method with detailed images, and it makes it possible to see the meniscus clearly. Tear meniscus change can be evaluated with TBUT test easily. [25-27] All tear meniscus parameters, including TMA, TMH and TMD, of the depression and anxiety 
groups were significantly lower compared with the control group. As far as we know, there was no study that had evaluated the DED in mood disorders with AS-OCT. Although, conventional DED diagnose tests are minimally invasive, anxiety of the test during application may affect the results, especially in anxiety patients. Therefore, the non-invasive nature of this method became important for these patients.

Similar to previous studies we also examined the correlation between DED tests and BDI and BAI. [15, 23, $24,28]$ Previous studies did not report a correlation between these parameters, but our study BAI score was correlated with TBUT in patients with anxiety while BDI was correlated with TBUT, TMD, TMH and TMA in patients with depression. Taken together, these demonstrate the effects of these diseases itself on ocular surface without any medication. In fact, all previous studies were designed with the patients who were already on psychiatric medication, except for one. [15]

One of the limitations of this study was the small sample size per group and the status for DED and psychiatric problems may vary by day in each participant. Next, we were unable to follow patients for dry eye, after they were started on psychiatric medications, therefore we do not know the effects of these medications.

\section{Conclusions}

In conclusion, we found in our study a strong association between DED and newly diagnosed depression and anxiety. In addition, there were correlations between inventory scores and DED test values. We suggest that ophthalmological examination may be required before starting the patient on psychiatric medication.

\section{Acknowledgements}

Not applicable.

\section{Availibility of data and materials}

Not applicable.

\section{Author's contributions}

SIU performed psychiatric evaluation of the participants. MOU performed ophthalmological evaluation of the participants. MOU and SAK, analyzed and interpreted the data and they are major contributor in writing the manuscript. All authors read and approved the final manuscript.

\section{Funding}

The study was supported by Başkent University Research Fund

\section{Ethics approval and consent to participate}

This cross-sectional study adhered to the tenets of the Declaration of Helsinki. This study was approved by Institutional Review Board and Ethics Committee of the Baskent University (Project no: KA17/106).

\section{Consent for publication}

Informed consent was obtained from all patients.

\section{Competing interests}

The authors declare that they have no competing interests.

\section{Author details}

'Department of Ophthalmology, Başkent University School of Medicine Konya Research Hospital, 42000 Konya, Turkey. ²Department of Psychiatry, Başkent University School of Medicine Konya Research Hospital, Konya, Turkey. ${ }^{3}$ Department of Ophthalmology, Uludağ University School of Medicine, Bursa, Turkey.

Received: 7 January 2019 Accepted: 19 July 2019

Published online: 09 August 2019

\section{References}

1. Wen W, Wu Y, Chen Y, Gong L, Li M, Chen X, et al. Dry eye disease in patients with depressive and anxiety disorders in Shanghai. Cornea. 2012; 31(6):686-92.

2. The definition and classification of dry eye disease: report of the Definition and Classification Subcommittee of the International Dry EyeWorkShop (2007). Ocul Surf. 2007;5(2):75-92

3. Roh HC, Lee JK, Kim M, Oh JH, Chang MW, Chuck RS, et al. Systemic Comorbidities of Dry Eye Syndrome: The Korean National Health and Nutrition Examination Survey V, 2010 to 2012. Cornea. 2016;35(2):187-92.

4. Wang TJ, Wang IJ, Hu CC, Lin HC. Comorbidities of dry eye disease: a nationwide population-based study. Acta Ophthalmol. 2012:90(7):663-8.

5. Koçer $E$, Koçer A, Özsütçü M, Dursun AE, Krpnar I. Dry Eye Related to Commonly Used New Antidepressants. J Clin Psychopharmacol. 2015; 35(4):411-3

6. Chia EM, Mitchell P, Rochtchina E, Lee AJ, Maroun R, Wang JJ. Prevalence and associations of dry eye syndrome in an older population: the Blue Mountains Eye Study. Clin Exp Ophthalmol. 2003;31(3):229-32.

7. Apostol S, Filip M, Dragne C, Filip A. Dry eye syndrome. Etiological and therapeutic aspects. Oftalmologia. 2003;59(4):28-31.

8. Schiffman RM, Christianson MD, Jacobsen G, Hirsch JD, Reis BL. Reliability and validity of the ocular surface disease index. Arch Ophthalmol. 2000; 118(5):615-21.

9. Lu P, Chen X, Liu X, Yu L, Kang Y, Xie Q, et al. Dry eye syndrome in elderly Tibetans at high altitude: a population-based study in China. Cornea. 2008; 27(5):545-51.

10. Lin PY, Tsai SY, Cheng CY, Liu JH, Chou P, Hsu WM. Prevalence of dry eye among an elderly Chinese population in Taiwan: the Shihpai eye study. Ophthalmology. 2003:110(6):1096-101.

11. Skre I, Onstad S, Torgersen S, Kringlen E. High interrater reliability for the Structured Clinical Interview for DSM-III-R Axis I (SCID-I). Acta Psychiatr Scand. 1991;84(2):167-73.

12. Beck AT, Steer RA. Beck anxiety inventory manual. San Antonio: The psychological Corporation; 1993.

13. Beck AT, Ward CH, Mendelson M, Mock J, Erbaugh J. An inventory for measuring depression. Arch Gen Psychiatry. 1961:4:561-71.

14. Bron AJ, Evans VE, Smith JA. Grading of corneal and conjunctival staining in the context of other dry eye tests. Cornea. 2003:22(7):640-50.

15. Tiskaoglu NS, Yazıcı A, Karlıdere T, Sari E, Oguz EY, Musaoglu M, et al. Dry eye disease in patients with newly diagnosed depressive disorder. Curr Eye Res. 2017:42(5):672-6.

16. Sharif NA, Senchyna M. Serotonin receptor subtype mRNA expression in human ocular tissues, determined by RT-PCR. Mol Vis. 2006;12:1040-7.

17. Chhadva P, Lee T, Sarantopoulos CD, Hackam AS, McClellan AL, Felix ER, et al. Human tear serotonin levels correlate with symptoms and signs of dry eye. Ophthalmology. 2015;122(8):1675-80

18. Ohayon MM, Schatzberg AF. Using chronic pain to predict depressive morbidity in the general population. Arch Gen Psyhiatry. 2003;60(1):39-47.

19. Croft PR, Papergeorgiou AC, Ferry S, Thomas E, Jayson MI, Silman AJ. Psychologic distress and low back pain: evidence from a prospective study in the general population. Spine (Phila Pa 1976). 1995:20(24):2731-7.

20. King SA. Pain Disorders. In: Hales RE, Yudofsky SC, Talbott JA, editors. Textbook of psychiatry. Washington: American Psychiatric Press; 1999. p. 1003-24.

21. Sarac H, Markeljevic J, Mokrovic G, Erdeljic V, Bozina N, Cicin-Sain L. Platelet serotonin in primary Sjögren's syndrome: level and relation with disease activity. J Neuroimmunol. 2012:251(1-2):87-9.

22. Kim KW, Han SB, Han ER, Woo SJ, Lee JJ, Yoon JC, et al. Association between depression and dry eye disease in an elderly population. Invest Ophthalmol Vis Sci. 2011;52(11):7954-8. 
23. Dibajnia P, Mohammadinia M, Moghadasin M, Amiri MA. Tear film break-up time in bipolar disorder. Iran J Psychiatry. 2012;7(4):191-3.

24. Li M, Gong L, Sun X, Chapin WJ. Anxiety and depression in patients with dry eye syndrome. Curr Eye Res. 2011:36(1):1-7.

25. Czajkowski G, Kaluzny BJ, Laudencka A, Malukiewicz G, Kaluzny JJ. Tear meniscus measurement by spectral optical coherence tomography. Optom Vis Sci. 2012;89(3):336-42.

26. Demirok GS, Gürdal C, Saraç Ö, Ceran BB, Can I. Evaluating of tear meniscus parameters with optical coherent tomography in dry-eye patients. Turk J Ophthalmol. 2013;43:258-62.

27. Akiyama R, Usui T, Yamagami S. Diagnosis of dry eye by tear meniscus measurements using anterior segment swept source optical coherence tomography. Cornea. 2015;34(Suppl 11):S115-20.

28. Labbé A, Wang YX, Jie Y, Baudouin C, Jonas JB, Xu L. Dry eye disease, dry eye symptoms and depression: the Beijing Eye Study. Br J Ophthalmol. 2013;97(11):1399-403.

Ready to submit your research? Choose BMC and benefit from:

- fast, convenient online submission

- thorough peer review by experienced researchers in your field

- rapid publication on acceptance

- support for research data, including large and complex data types

- gold Open Access which fosters wider collaboration and increased citations

- maximum visibility for your research: over $100 \mathrm{M}$ website views per year

At $\mathrm{BMC}$, research is always in progress.

Learn more biomedcentral.com/submissions 